\title{
Spaltung von dl-Aminocapronsäure (= Norleucin) in die optisch-aktiven Komponenten mittels der Formylverbindung. Polypeptide, an deren Aufbau Aminocapronsäure beteiligt ist. Von
}

Emil Abderhalden, C. Froehlich und Dionys Fuchs.

(Aụs dem phyșiologischen Institute der Universität Halle a. S.) (Der Redaktion zugegangen am 28. Juni 1913.)

Der Befund einer dem Leucin isomeren Verbindung ${ }^{1}$ ) unter den Spaltprodukten vọn Eiweißstoffen, die am Aufbau des Nervengewebes beteiligt sind, hat uns veranlaßt, die außer der $\alpha$-Aminoisobutylessigsäure und der $\beta$-Methyl- $\beta$ äthyl- $\alpha$-aminopropionsäure noch möglichen Aminosäuren der allgemeinen Formel $\mathrm{C}_{6} \mathrm{H}_{13} \mathrm{NO}_{2}$ darzustellen und vor allem ihre Eigenschaften zu studieren. Wie der eine von uns (A.) ${ }^{1}$ ) mit Weil nachgewiesen hat, kommt der neuen Leucinart höchst wahrscheinlich die Struktur einer $\alpha$-Aminocapronsäure zu. Diese Verbindung ist bereits von Emil Fischer ${ }^{2}$ ) eingehend studiert worden. Er hat die synthetisch gewonnene dl-Verbindung mittels der Benzoylverbindung und deren Brucinsalz in die optisch-aktiven Komponenten zerlegt.

Wir haben zur Trennung der dl- $\alpha$-Aminocapronsäure in ihre beiden optisch aktiven Komponenten einen anderen, von Emil Fischer ${ }^{3}$ ) angegebenen und von ihm wiederholt benützten Weg eingeschlagen. Wir bereiteten die Formylverbindung der dl- $\alpha$-Aminocapronsäure und trennten dann die

1) Emil Abderhalden u. Arthur Weil, Diese Zeitschrift, Bd. 8:3, S. 425 (1913).

2) Emil Fischer, Berichte der Deutschen chem. Gesellsch., Bd. 33, S. 2370 (1900). - Emil Fischer und Rudolf Hagenbach, Ebenda, Bd. 34, S. 3764 (1901).

3) Emil Fischer und Otto Warburg, Berichte der Deutschen chem. Gesellsch., Bd. 38, S. 3997 (1905). 
beiden optisch-aktiven Komponenten durch Darstellung des Brucinsalzes in der bekannten Weise.

Wir haben die Darstellung der optisch-aktiven $\alpha$-Aminocapronsäuren vorgenommen, weil uns das Studium der Eigenschaften von Polypeptiden, an deren Aufbau diese Aminosäure beteiligt ist, aus verschiedenen Gründen erwünscht erschien. Einmal wird zu entscheiden sein, welche Polypeptide von bestimmten Fermenten zerlegt und welche nicht angegriffen werden. Auf diesem Wege wird man entscheiden können, welche von den beiden optisch-aktiven Formen der $\alpha$-Aminocapronsäure in der Natur vorkommt. Das bei der Hydrolyse von Nervengewebe aufgefundene Produkt dreht nach rechts. Es ist somit zu erwarten, daß jene Polypeptide, an deren Aufbau d- $\alpha$-Aminocapronsäure teilnimmt, dem fermentativen Abbau unterliegen, während solche Verbindungen, die unter den Bausteinen die l-Form der genannten Verbindung aufweisen, unabgebaut bleiben.

. Die $\alpha$-Aminocapronsäure ist, um zur Bildung der Namen für Polypeptide ein kurzes Wort zur Verfügung zu haben, als Caprin bezeichnet worden. Dieser Name ist jedoch mehrdeutig. Man würde ein Fett, an dessen Aufbau Caprinsäure beteiligt ist, auch Caprin nennen. Es sei deshalb der Name Caprin durch Norlencin ersetzt. Norleucin soll zum Ausdruck bringen, daß ein Leucin vorliegt, das die Struktur der normalen, in $\alpha$-Stellung substituierten Capronsäure besitzl.

Wir haben die folgenden Polypeptide gewonnen: Glycyl-d-norleucin, Glycyl-l-norleucin, Glycyl-dl-norleucin, dl-Leucyl-glycyl-dl-norleucin.

\section{Experimenteller Teil.}

Darstellung von dl-a-Aminocapronsäure.

$$
\mathrm{CH}_{3} \cdot \mathrm{CH}_{2} \cdot \mathrm{CH}_{8} \cdot \mathrm{CHH}_{8} \cdot \mathrm{CH} \cdot \mathrm{COOH} .
$$

$200 \mathrm{~g}$ käufliche Gärungscapronsäure wurden der fraktionierten Destillation unterworfen und der zwischen 195-2050 übergehende Teil aufgefangen. $188 \mathrm{~g}$ des Destillates erwärmten 
wir eine Stunde lang mit $26,3 \mathrm{~g}$ rotem Phosphor in einem Rundkolben am Wasserbade bei $50^{\circ}$. Dann wurden $661,7 \mathrm{~g}$ Brom tropfenweise zugegeben. Das Reaktionsprodukt erwärmten wir nach Beendigung dieser Operation noch eine Stunde lang im kochenden Wasserbade. Die Lösung wurde nunmehr unter kräftigem Turbinieren in $1000 \mathrm{ccm}$ heißen Wassers eingegossen und das abgeschiedene Öl mit Äther ausgeschüttelt. Nach dem Trocknen des Äthers wurde er abdestilliert und der Rückstand der fraktionierten Destillation unterworfen. Bei $17 \mathrm{~mm}$ Druck destillierte die $\alpha$-Bromcapronsäure zwischen $128-136^{\circ}$ über.

$195 \mathrm{ccm} \alpha$-Bromcapronsäure wurden mit der fünffachen Menge 25\% igen Ammoniaks versetzt und in den Brutschrank gestellt. Am nächsten Tage war die ganze Masse bereits krystallinisch erstarrt. Es wurde abgenutscht. Ausbeute 142,5 g. Die Mutterlauge wurde eingedampft, mit Ammoniak versetzt und wieder in den Brutschrank gestellt. Nach 2 tägigem Stehen erfolgte, nach Einengen der Flüssigkeit, wieder eine Krystallisation, welche durch Zugabe von Alkohol sich vervollständigte. Es ließen sich weitere $20,5 \mathrm{~g}$ a-Aminocapronsäure gewinnen. Nach einmaliger Umkrystallisation aus heißem Wasser war das Präparat rein und zeigte, im geschlossenen Kapillarrohr erhitzt, den richtigen Schmelzpunkt gegen $297-300^{\circ}$.

$0,1356 \mathrm{~g}$ Substanz gaben $0,2761 \mathrm{~g} \mathrm{CO}_{2}$ und $0,1226 \mathrm{~g} \mathrm{H}_{2} \mathrm{O}$.

Berechnet für $\mathrm{C}_{6} \mathrm{~N}_{13} \mathrm{NO}_{2}: 54,90 \% \mathrm{C}, 9,97 \% \mathrm{H}$. Gefunden: $55 ; 53 \% \mathrm{C}, 10,12 \% \mathrm{H}$.

Amino-N nach van Slyke: Ber. : 10,68\%, gef.: $11,12 \%$.

Darstellung der Formyl-d,1- $\alpha$-aminocapronsäure.

$159 \mathrm{~g} \mathrm{~d}, 1-\alpha$-Aminocapronsäure wurden mit der $11 / 2$ fachen Menge wasserfreier käuflicher Ameisensäure 3 Stunden lang auf dem Wasserbade erhitzt. Nun wurde das Lösungsmittel unter vermindertem Druck möglichst vollständig verdampft, der Rückstand wieder in Ameisensäure aufgenommen, 3 Stunden auf $100^{\circ}$ erhitzt, dann eingedampft und diese Operation noch

1) Hüfner, Journal f. prakt. Chemie, II. Folge, Bd. 1, S. 6 (1870). 
einmal wiederholt. Der feste Rückstand, der durch Absprengen des Kolbens gewonnen werden konnte, wurde zerkleinert, dann im Vakuumexșikkator über Kalihydrat von noch anhaftender Ameisensäure befreit und schließlich fein pulverisiert. Nun wurde, um die unveränderte Capronsäure zu entfernen, in kleinen Portionen mit der $1 \frac{1}{2}$ fachen Menge eiskalter n-Salzsäure rasch zerrieben, scharf abgenutscht, und mit wenig eiskaltem Wasser bis zur Chlorfreiheit gewaschen. Das Rohprodukt wurde unter Anwendung von Tierkohle aus möglichst wenig Wasser umkrystallisiert. Ausbeute $90 \mathrm{~g}$.

Die Formyl-d-l- $\alpha$-aminocapronsäure krystallisiert in glänzenden Nadeln. Beim Erhitzen im offenen Kapillarrohr erweicht die Substanz bei $110-111^{\circ}$ und schmilzt bei $114^{\circ}$ (unkorr.).

$0,2178 \mathrm{~g}$ Substanz verbrauchten bei der N-Bestimmung nach Kjeldahl 13,39 cem n/10-n-Schwefelsäure.

Gefunden: $N=8,62 \%$. Berechnet: $8,80 \%$.

Spaltung der Formyl-dl- $\alpha$-aminocapronsäure mit Brucin.

$50 \mathrm{~g}$ Formyl-dl- $\alpha$-aminocapronsäure wurden in 2 Liter absoluten Alkohols eingetragen und am Wasserbade erwärmt. Ein Teil der Substanz ging nicht in Lösung. Von diesem wurde abfiltriert. Es stellte sich bei der Prüfung heraus, daß der ungelöste Rückstand, der etwa $4,8 \mathrm{~g}$ wog, reine $\alpha$-Aminocapronsäure war. Die Formylgruppe war also teilweise abgespalten worden.

$112, \check{a} \mathrm{~g}$ wasserfreies Brucin wurden in $1600 \mathrm{ccm}$ absoluten Alkohols unter Erwärmen gelöst. Diese Lösung gaben wir zu der alkoholischen Lösung der Formyl-dl- $\alpha$-aminocapronsäure. Nach 12 stündigem Stehen in der Kälte begann die Abscheidung von Brucinsalz. Sie nahm nach energischem Reiben plötzlich stark zu. Nach weiteren 12 Stunden wurde die Krystallmasse scharf abgesaugt und mit kaltem absolutem Alkohol gewaschen.-Die Krystallmasse wog ca. $95 \mathrm{~g}$. Sie bestand aus dem Brucinsalz der Formyl-d- $\alpha$-aminocapronsäure.

Die Substanz wurde in $530 \mathrm{ccm}$ Wasser gelöst, auf $0^{\circ}$ abgekühlt und mit $158 \mathrm{ccm}$ n-Natronlauge versetzt. Es fiel 
das Brucin aus. Nach einer Viertelstunde wurde abgesaugt und der Rückstand mit wenig kaltem Wasser gewaschen. Die letzten Reste von Brucin wurden durch Ausschütteln mit Chloroform vollständig entfernt. Nun wurden $20 \mathrm{ccm} 5$ fach Normalsalzsäure zugegeben. Dann engten wir die Lösung unter vermindertem Druck ein, übersättigten mit weiteren $14 \mathrm{ccm} 5 \mathrm{fach} \mathrm{n-Salz-}$ säure und stellten das Gemisch in Eiswasser. Nach einer halben Stunde wurde die Krystallmasse abgenutscht und mit eiskaltem Wasser chlorfrei gewaschen. Ausbeute ca. 15 g. ${ }^{1}$ )

Die alkoholische Mutterlauge, welche das Brucinsalz der Formyl-1- $\alpha$-aminocapronsäure enthielt, wurde unter vermindertem Druck eingedampft, der Rückstand in Wasser aufgenommen und weiter so behandelt, wie die d-Verbindung. Ausbeute ca. $15 \mathrm{~g}$.

Beide optisch-aktiven Formylkörper wurden aus wenig heißem Wasser umkrystallisiert, abgesaugt, und mit wenig kaltem Wasser gewaschen. Um die Abspaltung der Formylgruppe möglichst zu beseitigen, wurden die Substanzen in einem Exsikkator über Schwefelsäure getrocknet, welches in einem Gefäß wasserfreie Ameisensäure enthielt.

Die beiden optisch-aktiven Antipoden haben bis auf die Drehung völlig übereinstimmende Eigenschaften. Sie krystallisieren in feinen seidenglänzenden, kürzeren oder längeren Nadeln, welche zu büschelförmigen Gruppen angeordnet sind. Sie lösen sich sehr leicht in Wasser, in Äthyl- und Methylalkohol und in Äther.

Beim Erhitzen im offenen Kapillarrohr erweichen beide bei 1110. Bei $114^{\circ}$ findet ein richtiges Schmelzen statt. erhalten:

Bei der optischen Untersuchung wurden folgende Werte

I. $0,3769 \mathrm{~g}$ Substanz in $9,7547 \mathrm{~g}$ wässeriger Lösung drehen im $2 \mathrm{dm}-\mathrm{Rohr}-0 ; 90,[\alpha]_{\mathrm{D}}^{20}=-15,85$.

II. $0,2121 \mathrm{~g}$ Substanz in $10,0124 \mathrm{~g}$ wässeriger Lösung drehen im $1 \mathrm{dm}-\mathrm{Rohr}+0,33,[\alpha]_{\mathrm{D}}^{20}=+15,53$.

1) Die Ausbeute läßt sich ohne Zweifel noch erheblich șteigern. 


\section{Analysen:}

I. Formyl-d- $\alpha$-aminocapronsäure $6,01 \mathrm{mg}$ Substanz gaben $11,62 \mathrm{mg} \mathrm{CO}$ und $4,38 \mathrm{mg} \mathrm{H}_{2} \mathrm{O}$. (Mikroanalyse nach Pregl.)

$0,2040 \mathrm{~g}$ Substanz verbrauchten $12,702 \mathrm{ccm} \mathrm{n} / 10-\mathrm{H}_{2} \mathrm{SO}_{4}$

Berechnet für $\mathrm{C}_{7} \mathrm{H}_{13} \mathrm{O}_{3} \mathrm{~N}$ (159) Gefunden

$$
\begin{array}{rrr}
\text { C } & 52,83 \% & 52,73 \% \\
\text { H } & 8,17 \% & 7,98 \% \\
\text { N } & 8,80 \% & 8,72 \%
\end{array}
$$

II. Formyl-l- $\alpha$-aminocapronsäure

$0,1450 \mathrm{~g}$ Substanz gaben $0,2830 \mathrm{~g} \mathrm{CO}_{2}$ und $0,1079 \mathrm{~g} \mathrm{H}_{2} \mathrm{O}$

\begin{tabular}{|c|c|c|}
\hline Berechnet für & $\mathrm{C}_{7} \mathrm{H}_{13} \mathrm{O}_{3} \mathrm{~N}$ (159) & Gefunden \\
\hline C & $52,83 \%$ & $53,22 \%$ \\
\hline $\mathrm{H}$ & $8,17 \%$ & $8,27 \%$ \\
\hline $\mathrm{N}$ & $8,80 \%$ & $8,61 \%$ \\
\hline
\end{tabular}
$0,2722 \mathrm{~g}$ Substanz verbrauchten nach $\mathrm{Kj}$ eld ahl $16,74 \mathrm{ccm}$ $\mathrm{n} / \mathrm{10}-\mathrm{H}_{2} \mathrm{SO}_{4}$

Wir haben auch die leichte Abspaltbarkeit der Formylgruppe studiert und zwar 1 . bei $37^{\circ}$ und 2. in der Siedehitze.

1. $0,1698 \mathrm{~g}$ Formyl- $d-\alpha$-aminocapronsäure wurden in Wasser gelöst. Das Gewicht der Lösung betrug $12,0317 \mathrm{~g}$. Die Lösung wurde in ein $1 \mathrm{dm}$-Rohr mit Wassermantel eingefüllt. Das Rohr kam in den Brutschrank. Es wurde in bestimmten Intervallen die Drehung der Lösung festgestellt. Die Daten sind in der folgenden Tabelle zusammengestellt.

Tabelle I.

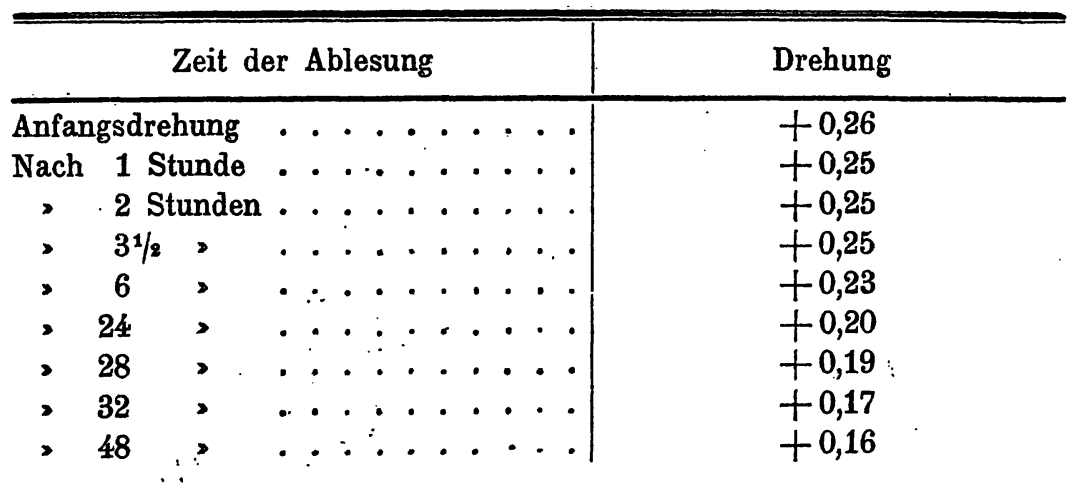


2. $0,5016 \mathrm{~g}$ Formyl-d- $\alpha$-aminocapronsäure wurden in $50 \mathrm{ccm}$ Wasser gelöst. Die Anfangsdrehung war im $2 \mathrm{dm}-\mathrm{Rohr}$ $+0,28$. Jetzt wurde 2 Stunden lang gekocht. Nach vollständigem Erkalten wurde die Lösung wieder auf das ursprüngliche Volumen gebracht und die Drehung wieder bestimmt. Dieselbe Operation wurde $4 \mathrm{mal}$ wiederholt. Die Daten sind aus der folgenden Tabelle zu ersehen.

Tabelle II.

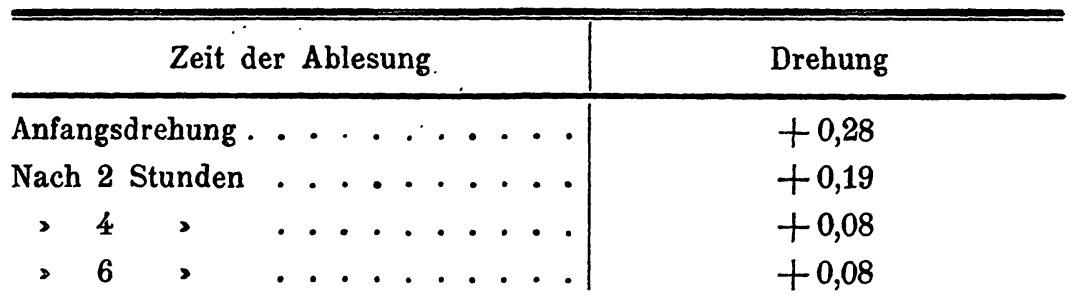

Darstellung der optisch-aktiven $\alpha$-Aminocapronsäuren.

Die optisch-aktiven $\alpha$-Aminocapronsäuren wurden durch Hydrolyse mit der. 10 fachen Menge 10\% iger Salzsäure aus den betreffenden Formylverbindungen dargestellt. Es wurde von $15 \mathrm{~g}$ der Formylkörper ausgègangen. Nach 1/2 stündigem Kochen am Rückflußkühler wurde die Lösung im Vakuum eingedampft, der Rückstand in Wasser aufgenommen und wieder eingedampft. Nun wurde der Rückstand in Wasser gelöst, in einen $500 \mathrm{ccm}-M e ß k o l b e n$ gebracht, dieser bis zur Marke aufgefüllt und in $1 \mathrm{ccm}$ der Lösung der Chlorgehalt nach Volhard bestimmt. Dann wurde die berechnete-Menge einer n-Lithiumhydroxydlösung zugegeben und das Gemisch in einer Porzellanschale am Wasserbade eingeengt. Es erfolgte Krystallisation, welche durch Zugabe von Alkohol sich vervollständigte. Sie wurde abgesaugt, mit kaltem Wasser und kaltem Alkohol chlorfrei gewaschen und mit wenig Tierkohle aus heißem Wasser umkrystallisiert. Ausbeute: 9 bezw. $8 \mathrm{~g}$.

Die beiden optisch-aktiven $\alpha$-Aminocapronsäuren krystallisieren in glänzenden schuppenförmigen Blättchen. Sie sind sehr schwer löslich in Wasser und Äthylalkohol. Beim Erhitzen im offenen Kapillarrohr sintert die Substanz bei 275 bis $280^{\circ}$ sehr stark und sublimiert teilweise weg. Bei $301^{\circ}$ 
beobachtet man deutliches Schmelzen, doch ist bis zum Erreichen dieser Temperatur der weitaus größte Teil der Substanz sublimiert.

Die Bestimmung des optischen Drehungsvermögens ergab die folgenden Werte. Es sei bemerkt, daß aus der d-Formyl$\alpha$-aminocapronsäure die l- $\alpha$-Aminocapronsäure und von der l-Formylverbindung die d- $\alpha$-Aminocapronsäure sich gebildet. Die optisch-aktiven $\alpha$-Aminocapronsäuren drehen sowohl in wässeriger Lösung, wie in 20\%iger Salzsäurelösung in derselben Richtung.

$$
\text { Spaltung I. }
$$

I. $0,1079 \mathrm{~g}$ Substanz in $17,8865 \mathrm{~g}$ wässeriger Lösung drehen im $2 \mathrm{dm}$-Rohr $-0,05^{01}$ )

$$
[\alpha]_{\mathrm{D}}^{200}=-4,14^{\circ} .
$$

II. 0,0842 g Substanz in $11,1026 \mathrm{~g}$ wässeriger Lösung drehen im $2 \mathrm{dm}-$ Rohr $+0,095^{\circ}$ )

$$
[\alpha]_{\mathrm{D}}^{20^{\circ}}=+6,26^{\circ} .
$$

I. $0,1080 \mathrm{~g}$ Substanz in $10,0693 \mathrm{~g} 20 \%$ iger Salzsäurelösung (spez. Gew. = 1,11) drehen im $2 \mathrm{dm}$-Rohr $-0,52$

$$
[\alpha]_{\mathrm{D}}^{200}=-21,17^{\circ}
$$

auf HCl-freie Substanz umgerechnet:

$$
[\alpha]_{D}^{200}=-17,00^{\circ} \text {. }
$$

II. $0,0720 \mathrm{~g}$ Substanz in $10,1526 \mathrm{~g} 20 \%$ iger HCl-Lösung (spez. Gew. $=1,11$ ) drehen im $2 \mathrm{dm}-\mathrm{Rohr}+0,28^{\circ}$

$$
[\alpha]_{\mathbb{D}}^{200}=+18,56^{\circ}
$$

auf HCl-freie Substanz umgerechnet:

$$
[\alpha]_{\mathrm{D}}^{200^{\circ}}=+15,6^{\circ} .
$$

Spaltung II.

I. $0,1268 \mathrm{~g}$ Substanz in $13,7940 \mathrm{~g}$ wässeriger Lösung drehen im $2 \mathrm{dm}-\mathrm{Rohr}+0,095^{1}$ )

$$
[\alpha]_{D}^{200}=+5,16^{\circ} \text {. }
$$

II. $0,1597 \mathrm{~g}$ Substanz in $16,4970 \mathrm{~g}$ wässeriger Lösung drehen im $2 \mathrm{dm}-\mathrm{Rohr}-0,087^{1}$ )

$$
[\alpha]_{D}^{20^{\circ}}=-4,49^{\circ}
$$

1) Mittelwerte aus $\cdot$ mehreren Ablesungen. 
462 Emil Abderhalden, C. Froehlich und Dionys Fuchs,

I. $0,1120 \mathrm{~g}$ Substanz in $11,3896 \mathrm{~g} 20 \%$ iger HCl-Lösung (spez. Gew. $=1,11$ ) drehen im $2 \mathrm{dm}$-Rohr $+0,45^{\circ}$

$$
[\alpha]_{D}^{200}=+20,44^{\circ}
$$

auf die HCl-freie Substanz umgerechnet:

$$
[\alpha]_{\mathrm{D}}^{20^{\circ}}=+16,02^{\circ} \text {. }
$$

II. $0,1360 \mathrm{~g}$ Substanz im 11,7498 g 20\% iger HCl-Lösung (spez. Gew. $=1,11$ ) drehen im $2 \mathrm{dm}-$ Rohr $-0,54^{\circ}$

$$
[\alpha]_{\mathrm{D}}^{200}=-20,82^{\circ}
$$

auf die HCl-freie Substanz umgerechnet:

$$
[\alpha]_{\mathrm{D}}^{200}=-16,07^{\circ} \text {. }
$$

Die d- $\alpha$-Aminocapronsäure schmeckt fad süß, die l- $\alpha$-Aminocapronsäure hat einen bitteren Geschmack.

Emil Fischer fand bei der Spaltung von dl-Aminocapronsäure mittels der Benzoylverbindungen die folgenden Werte für $[\alpha]_{20^{0}}^{\mathrm{D}}$ :

$$
[\alpha]_{D}^{20}=-22,4^{\circ} \text { und }[\alpha]_{D}^{20}=+21,3^{\circ} \text {. }
$$

Chloracetyl-d-norleucin.

$$
\mathrm{CH}_{3} \cdot \mathrm{CH}_{2} \cdot \mathrm{CH}_{2} \cdot \mathrm{CH}_{2} \cdot \underset{\mathrm{NH}}{\mathrm{CH}} \cdot \mathrm{COOH} \cdot(\mathrm{Cl}) \cdot \mathrm{CH}_{2} .
$$

$2,4 \mathrm{~g} \mathrm{~d}$ - $\alpha$-Aminocapronsäure wurden in $18,5 \mathrm{ccm}(1 \mathrm{Mol}$.) normaler Natronlauge gelöst und zu dieser Lösung 2,5 g (1,25 Mol.) Chloracetylchlorid und 27,7 ccm (1,5 Mol.) normaler Natronlauge im Laufe von $1 / 2$.Stunde in 10 Portionen unter Kühlung zugegeben. Nach Zufügen von $5,54 \mathrm{ccm}$ fünffach normaler Salzsäure schied sich ein kleine Krystalle enthaltendes dickes Öl aus. Dieses wurde in eine Kältemischung gestellt. Es erstarrte allmählich. Zur Reinigung wurde die amorphe Masse in Essigäther gelöst, die Lösung mit Tierkohle entfärbt und filtriert. Das Filtrat ließen wir bei gewöhnlicher Temperatur langsam eindunsten. Es schieden sich durchsichtige, lamellenförmig angeordnete Krystalle ab. Sie wurden scharf abgesaugt und wieder in Essigäther gelöst. Die Lösung wurde durch Verdampfen der Lösung fraktioniert krystallisiert. Die einzelnen 
Krystallfraktionen wurden abgesaugt, mit wenig absolutem Äther gewáschen und im Vakuumexsikkator über Schwefelsäure getrocknet. Ausbeute: $1,108 \mathrm{~g}$.

Beim Erhitzen im offenen Kapillarrohr wird die Substanz bei $70^{\circ}$ weich und schmilzt bei $104-106^{\circ}$ (unkorr.). Sie krystallisiert in farblosen, durchsichtigen Lamellen. Unter dem Mikroskop sieht man lanzettförmige, rhomboide Krystalle.

0,2444 g Substanz verbrauchten bei der Stickstoffbestimmung nach Kjeldảhl 11,702 ccm 1/10-n-Schwefelsäure.

Berechnet für $\mathrm{C}_{8} \mathrm{H}_{14} \mathrm{O}_{3} \mathrm{NCl}(207,5) \quad$ Gefunden

$$
\text { N. } \quad 6,76 \% \quad 6,70 \%
$$

$0,1752 \mathrm{~g}$ Substanz in $20,7724 \mathrm{~g}$ wässeriger Lösung drehen im $1 \mathrm{dm}-\mathrm{Rohr}+0,03,[\alpha]_{\mathrm{D}}^{200}=+3,56^{\circ}$.

$$
\begin{gathered}
\text { Glycyl-d-norleucin. } \\
\mathrm{CH}_{2} \cdot \mathrm{CH}_{2} \cdot \mathrm{CH}_{2} \cdot \mathrm{CH}_{2} \cdot \mathrm{CH} \cdot \mathrm{COOH} \\
\text { I } \mathrm{NH} \cdot \mathrm{CO} \cdot\left(\mathrm{NH}_{2}\right) \cdot \mathrm{CH}_{2} \cdot
\end{gathered}
$$

Es wurde das Chloracetyl-d-norleucin in bekannter Weise mit 25\%-igem wässerigem Ammoniak amidiert. Nach dreitägigem Stehen im Brutschrank wurde die Lösung unter vermindertem Druck eingedampft, der Rückstand in Wasser aufgenommen, mit Tierkohle entfärbt und die auf ein kleines Volumen eingedampfte Lösung mit viel Alkohol gefällt. Es entstand ein Niederschlag, der nach längerem Stehen in der Kälte und nach häufigem Reiben allmählich krystallinisch erstarrte. Der Krystallbrei wurde abgesaugt und der Rückstand in ganz wenig Wasser gelöst und wieder mit absolutem Alkohol gefällt. Diesmal fiel die Substanz schön krystallinisch aus. Sie wurde abgenutscht, mit wenig kaltem absolutem Alkohol gewaschen und im Vakuumexsikkator über Schwefelsäure getrocknet. Durch Einengen der Mutterlauge auf dem Wasserbade ließen sich noch weitere Mengen des Dipeptids gewinnen. Ausbeute $1,05 \mathrm{~g}$.

$0,1121 \mathrm{~g}$ Substanz verbrauchten bei der Stickstoffbestimmung nach Kjeldahl 12,135 ccm 1/10-n-Schwefelsäure. Daraus ergibt sich für Gly.cyl-d- $\alpha$-norleucin $N=15,155 \%$ (berechnet $14,89 \%$ ). 
464 Emil Abderhalden, C. Froehlich und Dionys Fuchs,

Mikroanalyse nach Pregl: 4,196 mg Substanz gaben $7,825 \mathrm{mg} \mathrm{CO}$ und $3,18 \mathrm{mg} \mathrm{H}_{2} \mathrm{O}, 2,176 \mathrm{mg}$ [716 mm, 21,5’] $0,297 \mathrm{ccm} \mathrm{N}$.

Berechnet für $\mathrm{C}_{8} \mathrm{H}_{16} \mathrm{O}_{3} \mathrm{~N}_{2}$ (188)

$$
\begin{array}{lr}
\text { Getü } \mathrm{C}_{8} \mathrm{H}_{16} \mathrm{O}_{3} \mathrm{~N}_{2}(188) & \text { Gefunden: } \\
\mathrm{C}=51,06 \% & 50,86 \% \\
\mathrm{H}=8,51 \% & 8,48 \% \\
\mathrm{~N}=14,89 \% & 15,15 \% \text { und } 14,95 \%
\end{array}
$$

Beim Erhitzen im offenen Kapillarrohr bräunt sich die Substanz bei $220^{\circ}$, sie sintert bei $230^{\circ}$ und schmilzt bei 239 bis $240^{\circ}$. Beim Schmelzen wurde die Substanz ganz schwarz. Das Glycyl-d-norleucin krystallisiert in Prismen, welche zu langen Nadeln ausgewachsen und teilweise an einem Ende zusammengewachsen sind.

0,0934 g Substanz in 10,1789 $\mathrm{g}$ wässeriger Lösung drehẹn im $2 \mathrm{dm}$-Rohr $-0,16$. Daraus berechnet $[\alpha]_{\mathrm{D}}^{20^{\circ}}=-8,71^{\circ}$.

$$
\begin{gathered}
\text { Glycyl-1-norleucin. } \\
\mathrm{CH}_{3} \cdot \mathrm{CH}_{2} \cdot \mathrm{CH}_{2} \cdot \mathrm{CH}_{2} \cdot \underset{\mathrm{CH}}{\mathrm{CH}} \mathrm{COOH} \\
\ldots \quad \mathrm{NH} \cdot \mathrm{CO} \cdot\left(\mathrm{NH}_{2}\right) \cdot \mathrm{CH}_{2} \cdot
\end{gathered}
$$

$3 \mathrm{~g} \mathrm{l}$ - $\alpha$-Aminocapronsäure wurden in $23,1 \mathrm{ccm}$ (1 Mol.) Normalnatronlauge gelöst und zu dieser Lösung 3,24 g (1,25 Mol.) Chloracetylchlorid und 37,6 ccm (1,5 Mol.) Normalnatronlauge im Laufe von $1 / 2$ Stunde in 10 Portionen zugegeben. Nach Zufügung der ersten Portionen bildete sich ein flockiger Niederschlag, welcher aber im weiteren Verlauf der Operation sich teilweise allmählich auflöste und teilweise sich in ein Öl umwandelte. Nun wurden $6,92 \mathrm{ccm}$ fünffach normale Salzsäure zugegeben. Das ausfallende Öl wurde mit Äther ausgeschüttelt und die abgetrennte Flüssigkeit im Vakuum eingedampft. Der Rückstand wurde zuerst mit heißem Äther und dann mit heißem Essigäther erschöpfend extrahiert. Alle Extrakte wurden vereinigt, mit geglühtèm Magnesiumsulfat getrocknet, abfiltriert und im Vakuum eingedampft. Das zurückbleibende Öl wurde in eine Krystallisationsschale gebracht. Es wurde in der verschiedensten Weise versucht, dieses Öl in Krystalle umzuwandeln, jedoch vergeblich. Offenbar war das Präparat nicht ganz rein. Wir verzichteten schließlich auf die Isolierung des Chloracetyl-l- 
norleucins und amidierten das Öl direkt. Die Ausbeute an Glycyl$\mathrm{d}$-norleucin betrug $1,8 \mathrm{~g}$.

$0,1227 \mathrm{~g}$ Substanz verbrauchten bei der Stickstoffbestimmung nach Kjeldahl 13,058 ccm ${ }^{1 / 10}$-n-Schwefelsäure. Daraus ergibt sich für Glycyl-l- $\alpha$-aminocapronsäure $\mathrm{N}=14,90 \%$ (berechnet $14,89 \%$ ).

Mikroanalyse nach Pregl: 4,407 mg Substanz gaben $8,27 \mathrm{mg}$ $\mathrm{CO}_{2}$ und 3,34 $\mathrm{mg} \mathrm{H}_{2} \mathrm{O}, 3,092 \mathrm{mg}\left(718 \mathrm{~mm}, 21^{\circ}\right) 0,413 \mathrm{ccm} \mathrm{N}$.

Berechnet für $\mathrm{C}_{8} \mathrm{H}_{16} \mathrm{O}_{3} \mathrm{~N}_{2}$ (188): Gefunden:

$$
\begin{array}{lc}
\text { C. } 51,06 \% & 51,18 \% \\
\text { H } 8,51 \% & 8,48 \% \\
\text { N } 14,89 \% & 14,90 \% \text { u. } 14,99 \%
\end{array}
$$

Beim Erhitzen im offenen Kapillarrohr bräunt sich die Substanz bei $220^{\circ}$, sie sintert bei $230^{\circ}$ und schmilzt bei 239 bis $240^{\circ}$. Beim Schmelzen wurde die Substanz ganz braun. Sie krystallisiert in Prismen, welche zu langen Nadeln ausgewachsen und teilweise an einem Ende zusammengewachsen sind.

$0,0979 \mathrm{~g} \mathrm{Substanz}$ in 10,0784 $\mathrm{g}$ wässeriger Lösung drehen im $2 \mathrm{dm}-\mathrm{Rohr}+0,16$. Daraus berechnet sich $[\alpha]_{\mathrm{D}}^{200}=+8,24$.

\section{Glycyl-dl-norleucin.}

$$
\text { Chloracetyl-dl-norleucin. }
$$

$$
\begin{array}{r}
\mathrm{CH}_{3} \cdot \mathrm{CH}_{2} \cdot \mathrm{CH}_{2} \cdot \mathrm{CH}_{2} \cdot \mathrm{CH} \cdot \mathrm{COOH} \\
\mathrm{NH} \cdot \mathrm{CO} \cdot(\mathrm{Cl}) \cdot \mathrm{CH}_{2} .
\end{array}
$$

$13,1 \mathrm{~g}$ Aminocapronsäure wurden in $100 \mathrm{ccm} \mathrm{n}-\mathrm{NaOH}-$ Lösung gelöst und in der Kälte abwechselnd mit $150 \mathrm{ccm}$ n-NaOH-Lösung und 11,5 ccm Chloracetylchlorid unter Schütteln versetzt. Die Operation dauerte $1 / 2$ Stunde. Von den sich in geringen Mengen abscheidenden Flocken wurde abfiltriert und das Filtrat zur Fällung der freien Verbindung mit $20 \mathrm{ccm}$ 5-n-Salzsäure tropfenweise versetzt, wobei sich die Substanz teilweise krystallinisch abschied. Nach 12 stündigem Stehenlassen in der Kälte wurde abfiltriert (die Mutterlauge kaun zur Gewinnung des Restes mit viel Äther extrahiert werden) und der Rückstand auf der Tonplatte getrocknet. Ausbeute $16 \mathrm{~g}$. 
466 Emil Abderhalden, C. Froehlich und Dionys Fuchs,

ZurReinigung wurde das Produkt in Aceton bei Zimmertemperatur gelöst und mit Wàsser bis zur bleibenden, schwachen Trübung versetzt. Die Verbindung schied sich beim Stehen in langen Prismen aus. Fp. 104-1070. Leicht löslich in Alkohol, Aceton, Äther, unlöslich in W asser.

$0,2450 \mathrm{~g}$ Substanz verbrauchten $11,85 \mathrm{ccm}^{1 / 10}$-n-Schwefelsäure.

Berechnet für $\mathrm{C}_{8} \mathrm{H}_{14} \mathrm{O}_{3} \mathrm{NCl}(207,5)$ :

Gefunden:

$6,76 \% \mathrm{~N}$

$6,77 \% \mathrm{~N}$.

$$
\begin{aligned}
& \text { Glycyl-dl-norleucin. } \\
& \mathrm{CH}_{3} \cdot \mathrm{CH}_{2} \cdot \mathrm{CH}_{2} \cdot \mathrm{CH}_{2} \cdot \mathrm{CH} \cdot \mathrm{COOH} \\
& \quad \mathrm{NH} \cdot \mathrm{OC} \cdot\left(\mathrm{NH}_{2}\right) \cdot \mathrm{CH}_{2} \cdot
\end{aligned}
$$

$12 \mathrm{~g}$ Chloracetyl-dl-norleucin wurden in $120 \mathrm{ccm} \mathrm{NH}_{3}(25 \%)$ gelöst, wobei die Verbindung beim Schütteln langsam in Lösung ging. Die Lösung wurde 3 Tage bei $37^{\circ}$ lang in einer verschlossenen Stöpselflasche aufbewahrt. Da sich im Laufe der Zeit keine Krystalle gebildet hatten, wurde die Lösung auf dem Wasserbade bis zur Trockne langsam eingedampft. Das Abdampfen wurde nach Zusatz von Alkohol und Wasser mehrmals wiederholt. Zur Trennung des Dipeptides vom Chlorammonium wurde der Rest mit wenig Wasser und Alkohol verrieben, und die hinterbliebene krystallinische Masse abfiltriert. Sie bestand fast ganz aus reinem Dipeptid. Ausbeute 8 g. Zur Reinigung wurde die Substanz in kochendem Wasser gelöst. Das Dipeptid fiel beim Abkühlen in Form von Blättchen aus. Sie fühlen sich fettig an. Fp. $210-215^{\circ}$; sintert bei $210^{\circ}$, zersetzt sich bei $215^{\circ}$. Eine kleine, verdünntere Probe gab nach längerem Stehen Büschel von Prismen mit dem gleichen F. 210-215 ${ }^{\circ}$. Das Dipeptid ist bei Zimmertemperatur in Wasser nur wenig löslich; mehr löslich in kochendem Wasser. Durch Zusatz von Alkohol kann die Substanz in besserer Ausbeute abgeschieden werden. säure.

$0,2245 \mathrm{~g}$ Substanz verbrauchten $24,40 \mathrm{ccm} 1 / 10-n-S c h w e f e l-$

$$
\begin{array}{cc}
\text { Berechnet für } \mathrm{C}_{8} \mathrm{H}_{16} \mathrm{O}_{3} \mathrm{~N}_{2}: & \text { Gefunden: } \\
14,89 \% \mathrm{~N} & 15,17 \% \mathrm{~N} .
\end{array}
$$




\section{dl-Lencyl-glycyl-dl-norlencin.}

$\alpha$-Bromisocapronyl-glycyl-dl-norleucin.<smiles>CCCC(NC(=O)CNC(=O)C(Br)CC(C)C)C(=O)O</smiles>

$3,74 \mathrm{~g}$ Glycyl-dl-norleucin wurden in $20 \mathrm{ccm} \mathrm{n-NaOH}-$ Lösung gelöst und in der Kälte unter Schütteln abwechselnd mit $30 \mathrm{ccm}$ n-NaOH-Lösung und $3,6 \mathrm{ccm}$ Bromisocapronylbromid versetzt. Dauer der Operation 1/2 Stunde. Von den geringen Mengen ausgeschiedenen Harzes wurde abfiltriert und das Filtrat mit $4 \mathrm{ccm} 5-\mathrm{n}-\mathrm{HCl}$ tropfenweise versetzt. Anfangs fällt das Bromid ölig aus, es wird beim Reiben mit dem Glasstab bald fest. . Nach dem Abfiltrieren und Trocknen auf der Tonplatte wurden $7 \mathrm{~g}$ feste Substanz erhalten. Das Rohprodukt hatte einen Schmelzpunkt gegen $100^{\circ}$. Zur Reinigung wurde die Substanz bei Zimmertemperatur in Aceton gelöst und bis zur Trübung mit Wasser versetzt, wobei nach längerem Stehen die Verbindung auskrystallisiert war. Blättchen vom F. $140^{\circ}$, sintert früher. Leicht löslich in Aceton, Alkohol und Äther. Unlöslich in Wasser und Petroläther.

0,2001 g Substanz verbrauchten 11,00 ccm 1/10-n-Schwefelsäure.

Berechnet für $\mathrm{C}_{14} \mathrm{H}_{25} \mathrm{O}_{4} \mathrm{~N}_{2} \mathrm{Br}: 7,67 \% \mathrm{~N}$. Gefunden: $7,69 \% \mathrm{~N}$.

dl-Leucyl-glycyl-dl-norleucin.<smiles>CC(C)CCN</smiles><smiles>CCCCCCCCCC(=O)NCC(=O)O</smiles>

$3,64 \mathrm{~g} \alpha$-Bromisocapronyl-glycyl-norleucin wurden in $12 \mathrm{ccm}$ Ammoniak $\left(25 \%\right.$ ) gelöst und bei $37^{\circ}$ drei Tage lang in der Stöpselflasche aufbewahrt. Da sich Krystalle nicht ausgeschieden hatten, wurde die Lösung auf dem Wasserbade langsam eingedampft, wobei sich krystallinische Häutchen bildeten. Nach völligem Eindampfen wurde der Rest mit etwas Wasser und 
468 Abderhalden, Froehlich u. Fuchs, Über dl-Aminocapronsäure.

Alkohol versetzt und nochmals eingedampft. Zur Trennung des Tripeptides vom Bromammonium wurde der Rest mit wenig Wasser und Alkohol verrieben und das unlösliche Tripeptid abfiltriert. Ausbeute $1,8 \mathrm{~g}$. Das Tripeptid ist auch in kochendem Wasser sehr wenig löslich. Zwecks Reinigung wurde das Tripeptid in viel kochendem Wasser gelöst, filtriert und die Lösung auf dem Wasserbade eingedampft, wobei sich die Verbindung in krystallinischen Häutchen abschied. F. $230-250^{\circ}$; sintert bei $220^{\circ}$, zersetzt sich bei $250^{\circ}$.

$0,1512 \mathrm{~g}$ Substanz verbrauchten $15,35 \mathrm{ccm}^{1 / 10-n-S c h w e-}$ felsäure.

Berechnet für $\mathrm{C}_{14} \mathrm{H}_{27} \mathrm{O}_{4} \mathrm{~N}_{3}: 13,95 \% \mathrm{~N}$.

Gefunden: $\quad 14,14 \% \mathrm{~N}$.

Wir haben versucht, Glycyl-dl-norleucin mit Hefemacerationssaft (nach Lebedew) zu spalten. 0,4220 g des Dipeptids wurden in $25 \mathrm{ccm}$ physiologischer Kochsalzlösung gelöst. Zu $7 \mathrm{ccm}$ dieser Lösung fügten wir $1 \mathrm{ccm}$ des Macerationssaftes. Die anfänglich optisch inaktive Lösung zeigte im Laufe von ca. 24 Stunden eine Drehung von - 0,05 ${ }^{\circ}$. Der Saft selbst blieb inaktiv, wie ein Kontrollversuch ergab.

Daß der Macerationssaft sehr wirksam war, bewies ein Versuch mit dl-Leucyl-glycin. 0,2160 g Dipeptid wurden in $25 \mathrm{ccm}$ physiologischer Kochsalzlösung gelöst. $7 \mathrm{ccm}$ der Lösung $+1 \mathrm{ccm}$ Macerationssaft drehten $-0,10^{\circ}$. Nach einer Stunde betrug die Drehung - 0,50 ${ }^{\circ}$.

Ein zweiter Versuch mit Glycyl-dl-norleucin ergab das gleiche Resultat. Bei Verwendung eines Gemisches von Pankreassaft und Darmsaft vom Hunde war ebenfalls das Auftreten einer Drehung zu beobachten. Es kann nach diesen Beobachtungen nicht entschieden werden, ob eine asymmetrische Spaltung des Dipeptids erfolgt ist. Die auftretende Drehung ist zu gering, als $\cdot \mathrm{da}$ sich ein bestimmter Schluß ziehen ließe. Die Schwerlöslichkeit des Dipeptids und die geringe Drehung der entsprechenden optisch-aktiven Dipeptide erschwert die Verfolgung der Spaltung auf dem optischen Wege. Aus dem gleichen Grunde ergab der Versuch, dl-Leucyl-glycyl-dl-norleucin mittels Hefemacerationssaftes zu spalten, kein eindeutiges Resultat. 